gr-qc/0312044

CERN-TH/2003-296

MIFP-03-26

\title{
Space-Time Foam may Violate the Principle of Equivalence
}

\author{
John Ellis $^{a}$, N.E. Mavromatos ${ }^{b, c}$, D.V. Nanopoulos ${ }^{d}$ and A.S. Sakharov ${ }^{a, e, f}$ \\ a CERN, Theory Division, CH-1211 Geneva 23, Switzerland. \\ ${ }^{b}$ King's College London, University of London, Department of Physics, Strand \\ WC2R 2LS, London, U.K. \\ ${ }^{c}$ Departamento de Física Téorica, Universidad de Valencia, E-46100, Burjassot, \\ Valencia, Spain. \\ ${ }^{d}$ George P. and Cynthia W. Mitchell Institute for Fundamental Physics, Texas \\ A\&M University, College Station, TX 77843, USA; \\ Astroparticle Physics Group, Houston Advanced Research Center (HARC), \\ Mitchell Campus, Woodlands, TX 77381, USA; \\ Academy of Athens, Academy of Athens, Division of Natural Sciences, \\ 28 Panepistimiou Avenue, Athens 10679, Greece. \\ e Swiss Institute of Technology, ETH-Zürich, 8093 Zürich, Switzerland. \\ ${ }^{f}$ INFN Laboratory Nazionali del Gran Sasso, SS. 17bis 67010 Assergi (L'Aquila), \\ Italy.
}

\begin{abstract}
The interactions of different particle species with the foamy space-time fluctuations expected in quantum gravity theories may not be universal, in which case different types of energetic particles may violate Lorentz invariance by varying amounts, violating the equivalence principle. We illustrate this possibility in two different models of space-time foam based on D-particle fluctuations in either flat Minkowski space or a stack of intersecting D-branes. Both models suggest that Lorentz invariance could be violated for energetic particles that do not carry conserved charges, such as photons, whereas charged particles such electrons would propagate in a Lorentz-inavariant way. The D-brane model further suggests that gluon propagation might violate Lorentz invariance, but not neutrinos. We argue that these conclusions hold at both the tree (lowest-genus) and loop (higher-genus) levels, and discuss their implications for the phenomenology of quantum gravity.
\end{abstract}




\section{Introduction}

The reconciliation of general relativity and quantum field theory in a true quantum theory of gravity remains elusive. Perhaps one or the other, or both, of these fundamental theories undergoes some modification when embedded in quantum gravity? A possible hint for a need to modify quantum field theory may have been provided by black-hole thermodynamics [1]. In general, information is lost across a horizon, such as that surrounding a black hole, which must therefore be described as a mixed quantum-mechanical state. However, one can imagine preparing a black hole from a pure quantum-mechanical initial state, so must we formulate [2] a theory that, unlike conventional quantum mechanics and quantum field theory, allows pure states to evolve into mixed states? Another suggestion is that Lorentz symmetry may be broken in a quantum theory of gravity. In such a theory, the vacuum presumably needs to be treated as a dynamical medium - a space-time foam containing many evanescent fluctuations. Energetic particles may induce recoil effects on structures in the foam, and these may, in return, induce back-reaction effects on the the particles' propagation. Do these cause energetic particles to travel at less than the conventional speed of light?

In this paper we propose a third possible signature of quantum gravity, namely a violation of the equivalence principle [3], in the sense that different types of ultrarelativistic particles may propagate in different ways. Within the general quantumgravitational framework outlined above, such equivalence breaking might arise if the back-reaction of the recoiling space-time foam is non-universal for different particle species. This would be unexpected if the structures that appear and disappear in the space-time foam have purely gravitational interactions with the propagating matter particles. However, there might also be non-gravitational interactions with structures in the space-time foam, which need not be universal. In this case the back-reactions might depend on the species of the propagating particle [4.

We demonstrate that just such a feature appears in a D-brane model of spacetime foam [5] that we have used previously to motivate the suggestion that energetic photons might travel at less than the standard velocity of light measured for lowenergy photons [6, 7]. We show that the same mechanism that slows down energetic photons, producing an energy-dependent in vacuo refractive index, is inoperative for relativistic particles carrying a conserved charge, such as electrons. This is because this approach models the structures in the space-time foam as D-particles, and neither they nor their possible excitations can carry electric charge.

We were led to this observation that the principle of equivalence might be violated for ultra-relativistic particles by considering the severe constraints on Lorentz 
violation for electrons [8, 4] that are imposed by the apparent observation of synchrotron radiation from the Crab Nebula 8]. We observed 4] that this impressive constraint does not apply to photons, and we then realized that our D-brane evades the Crab Nebula constraint just because it predicts Lorentz invariance for electrons, in contrast to photons [3].

In this paper, we focus on the explanation of this equivalence breaking in two variants of the D-particle model, one based on string propagation in Minkowski space and the other invoking intersecting stacks of D-branes [9]. The latter suggests that the propagation of energetic gluons might violate Lorentz invariance, but not that of energetic neutrinos. We argue that these conclusions should hold also at higher orders in the string genus (loop) expansion. Finally, we comment on the interpretation of the constraint on quantum gravity provided by synchrotron radiation from the Crab Nebula, and on other implications for quantum-gravity phenomenology.

\section{Space-Time Foam and the Violation of the Equiv- alence Principle}

It may at first sight seem paradoxical that space-time foam could violate the equivalence principle, since one expects gravity to exhibit universal properties at low energies. This low-energy universality is a well-established feature of string theory, but it is not guaranteed that this universality should extend to high energies. For example, in brane physics, excitations with non-zero charges, e.g., under the Standard Model gauge group, are represented by open strings with their ends attached with Dirichlet boundary conditions to a brane [9]. Only neutral, closed-string excitations are allowed to propagate in the bulk space transverse to the brane. This framework is manifestly non-universal in general.

We display the advertised non-universal violation of the equivalence principle for ultra-relativistic particles in two different ways in the following two subsections. The first demonstration is outside the brane-world framework, and the second is within it.

\subsection{D-Particle Model of Space-Time Foam}

In the original model of [5], one treats observable particles as closed-string states propagating through a 'bulk' space-time. Space-time foam as comprised of pointlike defects, each of which is modelled as a zero-space-dimensional D0-brane or D-particle, embedded in this four- (or higher-) dimensional bulk space-time, which 
(a)

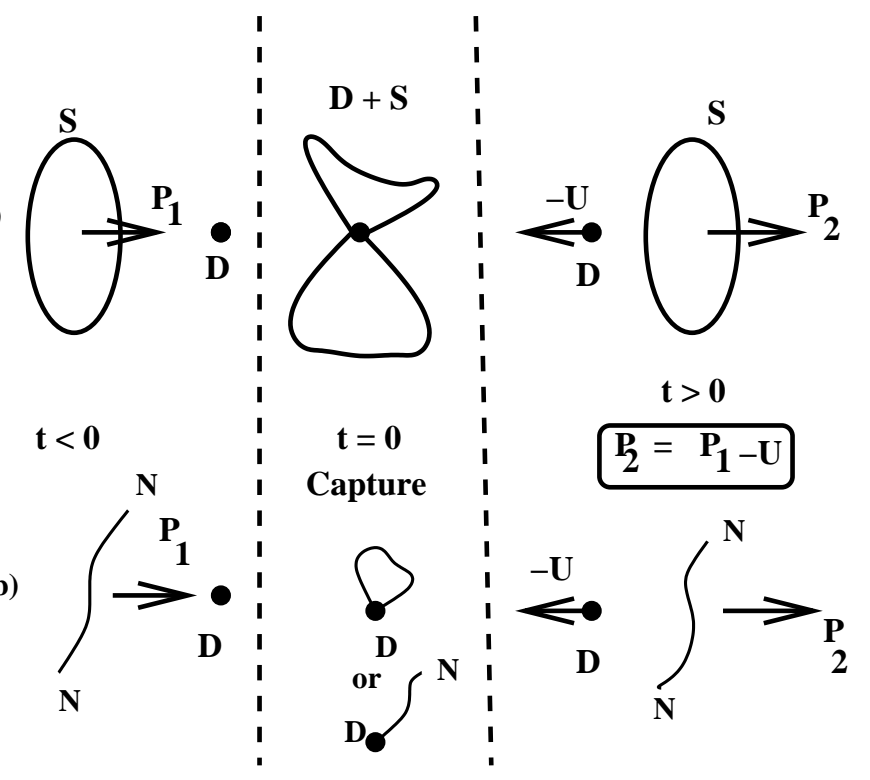

Figure 1: In the Liouville model of space-time foam [5], only a string particle (S) neutral under the (unbroken) Standard Model group can be captured by a D-particle defect $(D)$ in space time. This results in a modified (subluminal) dispersion relation for $S$, as a consequence of the recoil of the defect $D$ in a direction opposite to the incident beam. Drawing (a) shows the capture of a closed string $S$ by the defect, and drawing (b) shows the capture of an open string $S$ by the defect. All capture processes involve a change in the boundary conditions of the respective $\sigma$-model fields on open world sheets.

represents the Minkowski space-time we observe. An energetic particle propagating with momentum $p_{i}$ may strike a relatively heavy defect with mass $\sim M_{s}$, the string scale with a coupling $g_{s}$, causing it to recoil, as illustrated in Fig. 11 This process was discussed in detail in [5], where it was shown that the associated back-reaction effects in target space lead to the following energy-dependent modified effective metric 'felt' by the energetic particle:

$$
G_{00}=-1 ; \quad G_{i j}=\delta_{i j} ; \quad G_{0 i}=g_{s} \frac{\Delta p_{i}}{M_{s}} \sim \frac{1}{2} g_{s} \frac{p_{i}}{M_{s}} \sim \frac{p_{i}}{\mathcal{M}}
$$

where $\Delta p_{i}$ is the momentum transfer during the scattering process and $M_{s} / g_{s}=\mathcal{M}$ is the effective quantum-gravity scale [5]. 
In more detail, in the recoil/back-scattering process illustrated in Fig. 1, a closedstring (matter) state - we recall that in this model there are no open strings in the bulk - is momentarily captured by the defect. It then splits into two open-string excitations with their ends attached in the defect, which then recombine to emit a closed-string state. This will, in general, have a new momentum, with the defect itself recoiling in a direction opposite to that of the incident particle.

The corresponding target-space quantum dynamics was discussed in [10] from a world-sheet $\sigma$-model point of view, in which the Galilean-boosted D-particle has position

$$
Y_{i}\left(x^{0}\right)=Y_{i}+U_{i} x^{0}
$$

where we have assumed a non-relativistic heavy D-particle with velocity $U_{i}$. The mathematical formulation of the capture/recoil process involves non-trivial impulse operators, as discussed in [10]. One may incorporate such excitations in the $\sigma$ model path integral by considering the world-sheet genus expansion of a matrix $\sigma$ model. An analysis of the annulus amplitude reveals that there are logarithmic divergences arising from integrations over the modular parameter $q$ of the form $\int d q / q$ [11, 12], describing appropriate summations of world-sheet annulus diagrams. These divergences can be removed by replacing the velocity operator in (21) by $\lim _{\epsilon \rightarrow 0^{+}} U_{i} D\left(x^{0} ; \epsilon\right)$, where

$$
D\left(x^{0} ; \epsilon\right)=x^{0} \Theta\left(x^{0} ; \epsilon\right)
$$

and

$$
\Theta(s ; \epsilon)=\frac{1}{2 \pi i} \int_{-\infty}^{\infty} \frac{d q}{q-i \epsilon} \mathrm{e}^{i q s}
$$

is the regulated Heaviside step function, with $\Theta(s) \equiv \lim _{\epsilon \rightarrow 0^{+}} \Theta(s ; \epsilon)=0$ for $s<0$ and $\Theta(s)=1$ for $s>0$. The infinitesimal parameter $\epsilon$ regulates the ambiguous value of $\Theta(s)$ at $s=0$, and the integral representation (4) is used because $x^{0}$ is eventually elevated to a quantum operator.

When this velocity term is inserted into the boundary integral of the $\sigma$-model action, the $\epsilon \rightarrow 0^{+}$divergences arising from the regulated step function can be used to cancel the logarithmic divergences of the annulus amplitudes [11, 12. This relates the target-space regularization parameter $\epsilon$ to the world-sheet ultraviolet scale $\Lambda$ by 13

$$
\epsilon^{-2}=-2 \alpha^{\prime} \log \Lambda
$$

This new velocity operator is called the impulse operator [12, and has non-zero matrix elements between different states of the D-particle. It describes recoil effects from the emission or scattering of closed string states off the D-particle, and, in 
the impulse approximation, it ensures that (classically) the D-particle starts moving only at time $x^{0}=0$.

But this is not all that is required. The operator (3) on its own does not lead to a closed conformal algebra. Computing its operator-product expansion with the stress-energy tensor shows [13] that it is only the pair of operators $D\left(x^{0} ; \epsilon\right), C\left(x^{0} ; \epsilon\right)$, where

$$
C\left(x^{0} ; \epsilon\right)=\epsilon \Theta\left(x^{0} ; \epsilon\right)
$$

that define an algebra that is closed under the action of the world-sheet stressenergy tensor. They form a pair of logarithmic operators of the conformal field theory 14. Thus, in order to maintain conformal invariance of the world-sheet theory, one cannot work just with the operator (3), because (6) will be induced by conformal transformations. If we rescale the world-sheet cutoff

$$
\Lambda \rightarrow \Lambda^{\prime}=\Lambda \mathrm{e}^{-t / \sqrt{\alpha^{\prime}}}
$$

by a linear renormalization group scale $t$, then (15) induces a transformation

$$
\epsilon^{2} \rightarrow \epsilon^{\prime 2}=\frac{\epsilon^{2}}{1-4 \sqrt{\alpha^{\prime}} \epsilon^{2} t}
$$

and we find

$$
D\left(x^{0} ; \epsilon^{\prime}\right)=D\left(x^{0} ; \epsilon\right)+t \sqrt{\alpha^{\prime}} C\left(x^{0} ; \epsilon\right) \quad, \quad C\left(x^{0} ; \epsilon^{\prime}\right)=C\left(x^{0} ; \epsilon\right) .
$$

If we now modify the initial position of the D-particle to $\lim _{\epsilon \rightarrow 0^{+}} \sqrt{\alpha^{\prime}} Y_{i} C\left(x^{0} ; \epsilon\right)$, then this scale transformation will induce, by conformal invariance, a transformation of the velocities and positions as follows:

$$
U_{i} \rightarrow U_{i} \quad, \quad Y_{i} \rightarrow Y_{i}+U_{i} t
$$

i.e., a Galilean evolution of the D-particle in target space.

In order to incorporate properly the non-trivial dynamics of the D-particle, one must therefore consider, instead of (2), the recoil operator

$$
Y_{i}\left(x^{0}\right)=\lim _{\epsilon \rightarrow 0^{+}}\left(\sqrt{\alpha^{\prime}} Y_{i} C\left(x^{0} ; \epsilon\right)+U_{i} D\left(x^{0} ; \epsilon\right)\right) .
$$

The conformal algebra reveals that the operators (3) and (6) have the same conformal dimension 13

$$
\Delta_{\epsilon}=-\alpha^{\prime}|\epsilon|^{2} / 2
$$


which vanishes as $\epsilon \rightarrow 0^{+}$. For finite $\epsilon$, the operator (111) yields a deformation of the world-sheet action of conformal dimension $1-\alpha^{\prime}|\epsilon|^{2} / 2$. This describes a relevant deformation of the $\sigma$-model, and the resulting string theory is therefore non-critical. It follows from (10) that the corresponding $\mathrm{RGE} \beta$ functions are

$$
\beta_{Y_{i}}=\Delta_{\epsilon} Y_{i}+\sqrt{\alpha^{\prime}} U_{i} \quad, \quad \beta_{U_{i}}=\Delta_{\epsilon} U_{i}
$$

As the 'dressing' by the operators $C$ and $D$ is determined entirely by the temporal coordinate $x^{0}$, we identify this field as the Liouville field $\varphi$ [5]. Marginality of the deformation is then restored by taking the limit $\epsilon \rightarrow 0^{+}$. However, in view of (10), the world-sheet scale $\epsilon^{-2}$ itself is identified with the target time [15, 15], and hence one is forced to dress the impulse operator by the Liouville/time field to restore conformal invariance for any finite time. It is this procedure, as explained in the relevant literature [15, 5] that leads to gravitational back-reaction effects, and to an effective target-space metric of the form (11).

The basic technical steps of this analysis may be summarised as follows. (i) One writes the recoil boundary operator as a bulk operator in terms of a total world-sheet derivative $\partial$, and then dresses it with a Liouville field $\phi$. (ii) Since the $D$ operator is the leading contribution as $\epsilon \rightarrow 0^{+}$, the dressed operator becomes in this limit $\int_{\partial \Sigma} e^{\alpha \phi} \partial_{\beta}\left(U_{i} x^{0} \Theta\left(x^{0} ; \epsilon\right) \partial^{\beta} x^{i}\right)$, where $\beta=1,2$ is a world-sheet index, and $\alpha=\epsilon$ is the gravitational anomalous dimension, computed according to the rules of Liouville dressing [15, 5]. (iii) One integrates this formula partially, by applying the Stokes theorem on the world sheet, and then uses the representation $\Theta\left(x^{0} ; \epsilon\right) \sim e^{-\epsilon x^{0}}$, where only the zero mode of $x^{0}$ is used ${ }^{1}$. (iv) In this way, one obtains boundary contributions that are conformally invariant, as well as a bulk world-sheet contribution of the form:

$$
U_{i} \int_{\Sigma} \epsilon x^{0} \partial \phi \partial x^{i} e^{\epsilon\left(\phi-x^{0}\right)}
$$

(iv) The analysis of [10, 15] indicates that $\bar{U}_{i} \equiv U_{i} / \epsilon$ is an exactly marginal (scaleinvariant) $\sigma$-model coupling, that can be identified with the physical recoil velocity, associated with the momentum transfer during the capture process. (v) Taking into account the identification of the zero mode of $\phi \equiv x^{0}$, and recalling that one is working at large times $\epsilon^{2} x^{0} \sim 1$, one readily observes that (14) corresponds to a world-sheet interaction $\propto \partial t \partial x^{i}$, and hence to a modification of the target-space background metric of the form (11). Physically, this is due to the displacement of

\footnotetext{
${ }^{1}$ This is justified by a more rigorous analysis in [15] 10].
} 
the D-particle defect in the background space-time following the collision with the propagating particle.

It has been shown in [5] that such effects yield an effective Born-Infeld action for a massless gauge field, which is the T-dual of (11), corresponding to a gauge potential of the form:

$$
A_{0}=0 \quad \text { (gauge condition), } \quad \mathrm{A}_{\mathrm{i}}\left(\mathrm{x}^{0}\right)=\left(\epsilon \mathrm{Y}_{\mathrm{i}}+\mathrm{U}_{\mathrm{i}} \mathrm{x}^{0}\right) \Theta\left(\mathrm{x}^{0} ; \epsilon\right)
$$

The open strings on the D-particle correspond, after a T-duality transformation, to gauge excitations. We can now consider in this T-dual picture the propagation of a real gauge field, represented by either a closed or open string moving through a bulk space. A D-particle defect can be viewed as a particular background of the corresponding $\sigma$ model.

It has been argued in [16 that it is possible to consider composite objects in which only one end of the open string is attached to the D-particle, the other end being free in the bulk space. However, as explained in [17], a fundamental string cannot end on an isolated D-particle, because the latter does not support a world-volume charge. Such configurations are allowed only in the presence of a neighbouring $(p=8)$-brane, in which case the other end of the open string is attached to the brane. Such a configuration experiences zero force, which makes it an appropriate vacuum of string theory. We do not consider such configurations here.

Instead, by analogy with the capture by the defect of a closed string, as described above, we consider the possibility that both ends of the open string are suddenly attached to the D-particle. In the case of isolated D-particles, this corresponds to a composite object which transforms as an Abelian gauge excitation under the action of the $U(1)$ group. In our model of D-particle foam, the latter may then be identified with a different state of the photon, differing from the incident one by a phase in its wave function. In a similar manner, the collision with the open string leaves the defect also with a phase difference in its wave function.

However, the capture (or emission) of a closed- or open-string particle, propagating through the bulk, by (or from) a D-particle defect is forbidden for strings representing electrically-charged matter particles. This is because, during the capture stage, which lasts for a characteristic 'uncertainty time' of order the string scale, as seen in Fig. 1, an intermediate composite state is produced. According to the discussion above, this object transforms as a gauge field, i.e., in the adjoint representation of the gauge group, whereas a charged particle would in general transform according to some other representation of the group ${ }^{2}$.

\footnotetext{
${ }^{2}$ In the presence of supersymmetry, the fermionic partners of gauge fields would behave in the
} 
In simple terms, electric charge conservation prevents the charged particle capture by the defect. If the propagating closed-string probe carried electric charge, this could not be absorbed by the defect, as it is originally electrically neutral, as are its excitations. An excited intermediate state is allowed only for incident photons, not for any other Standard Model particles ${ }^{3}$. This argument certainly excludes electrons and other elementary charged probes from having modified dispersion relations in the model of [5]. The discussion of composite particles such as protons is more complicated but, to the extent that they contain uncharged constituents such as gluons, they might also be susceptible to the effects of the Liouville foam. We present in the next subsection a model of space-time foam in which gluon propagation might indeed be affected.

This example of the possible breakdown of the equivalence principle in the $\mathrm{Li}$ ouville model of quantum-gravitational space-time foam [5] is just one example how the Crab Nebula synchrotron radiation constraint 8, 4, may be evaded.

\subsection{D-Brane Model for Space-Time Foam}

In the modern context of the D-brane approach to gauge theories, gauge-field excitations are viewed, not as closed-string excitations propagating through bulk spacetime, but rather as open strings with their ends attached to a stack of $N$ Dp-branes. If these branes are parallel, one finds excitations transforming in the adjoint representation of $U(N)$ (for oriented strings) [19]. If there is a stack of $N_{1}$ parallel Dp-(super)branes interesecting at an angle $\alpha$ with another stack of $N_{2}$ parallel Dpbranes, the gauge group is $U\left(N_{1}\right) \otimes U\left(N_{2}\right)$, and in such cases it is possible to define chiral fermions that are localised at the interesection [20, which are open-string excitations transforming under the bi-fundamental representation of the product gauge group. In such a way, by arranging appropriately various stacks of higherdimensional D-branes one may construct a representation of the Standard Model at such a (3+1)-dimensional intersection. In this subsection, we re-examine the propagation of different Standard Model particles in such a D-brane construction.

In our approach to D-particle space-time foam we consider a situation in which the intersection of such stacks of D-branes is punctured by D-particle defects, as seen in the left drawing in Fig. 2. In such a case, the open strings localised on the brane may be captured by the defect, as illustrated in part (b) of Fig. 11. The important issue for us is: which particle species may be captured? As we have

same way as the gauge bosons themselves 18 .

${ }^{3}$ It might also be possible for some particles beyond the Standard Model, such as right-handed (sterile) neutrinos, of other particles which are neutral under the Standard Model gauge group. 

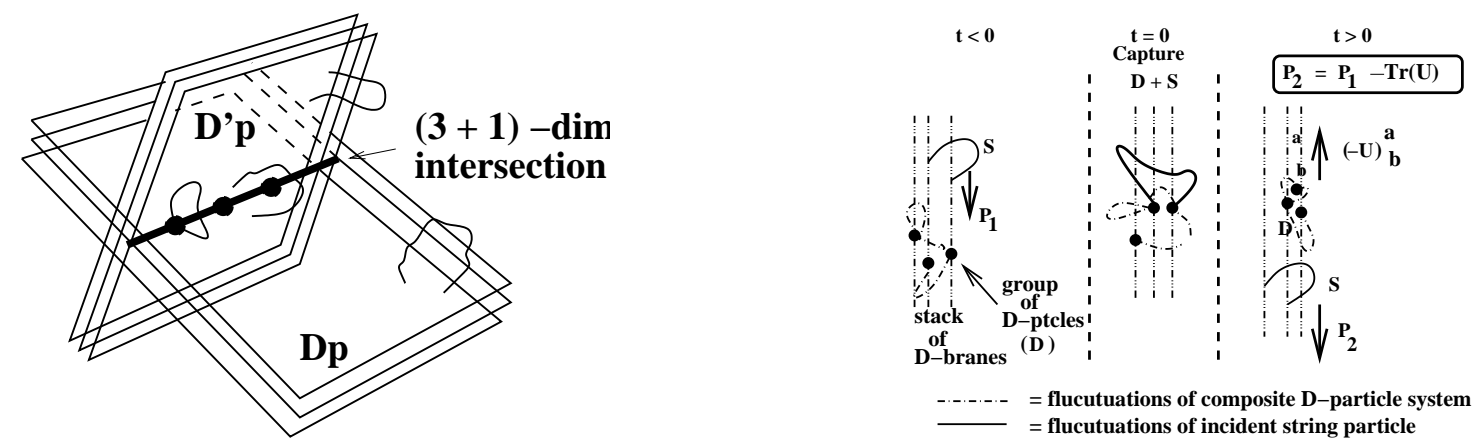

Figure 2: A model of space-time foam with recoil and back-reaction which preserves a non-Abelian gauge group. In the left drawing, the D-particles (dark blobs) can puncture the (3+1)-dimensional intersection of stacks of D-branes, and the foam effect is caused by their interactions with open-string states localised on the intersection. The right drawing shows a detailed view of these interactions on the $(3+1)$-dimensional stack of intersecting D-branes. A string mode with non-trivial non-Abelian $\mathcal{G}$ quantum numbers is represented as an open-string excitation of this stack of D-branes. Open strings can also stretch between the coincident D-particles (as shown by the dash-dotted lines between the dark blobs), describing excitations of groups of defects. The recoil and back-reaction dynamics of a group of $N$ interacting D-particles is described by massless gauge excitations of non-Abelian $U(N)$ Born-Infeld type. For virtual D-particle excitations, the group quantum numbers of the vacuum must be preserved, so $\mathcal{G} \simeq U(M)$ locally, imposing selection rules on the possible types of interaction with the foam.

mentioned above, in this $U\left(N_{1}\right) \otimes U\left(N_{2}\right)$ gauge theory living on the intersection, the chiral fermions in the bi-fundamental representation of the group and the gauge bosons in the adjoint representation manifest themselves very differently, and it is not obvious that any recoil/back-reaction effects should be universal.

In the case of string excitations with non-trivial quantum numbers under a nonAbelian gauge group, one is forced to consider more complicated foam configurations, involving groups of D-particle defects, interacting via the exchange of open strings stretching between them. The recoil/capture stage in such a model with non-trivial D-brane configurations has been studied in [10], where it was shown to be described by a non-Abelian gauge theory of Born-Infeld form.

To understand physically the situation, we recall that the central feature of Dbrane dynamics is the observation [19] that the low-energy effective field theory for 
a system of $N$ D-branes is ten-dimensional maximally supersymmetric $U(N)$ YangMills theory dimensionally reduced to the world-volume of the D-branes. For the case of D-particles, the Yang-Mills potential is

$$
V_{D 0}[Y]=\frac{\mathcal{T}^{2}}{4 g_{s}} \sum_{i, j=1}^{9} \operatorname{tr}\left[Y^{i}, Y^{j}\right]^{2}
$$

where $\mathcal{T}=1 / 2 \pi \alpha^{\prime}$ is the elementary string tension, $\alpha^{\prime}$ is the string Regge slope whose square root is the intrinsic string length $\ell_{s}$, and $g_{s}$ is the (dimensionless) string coupling constant. The fields $Y^{i}(t)$ are $N \times N$ Hermitian matrices in the adjoint representation and the trace is taken in the fundamental representation of the gauge group $U(N)$. In the free-string limit $g_{s} \rightarrow 0$, the field theory involving the potential (16) localizes onto those matrix configurations satisfying

$$
\left[Y^{i}, Y^{j}\right]=0 \quad, \quad i, j=1, \ldots, 9
$$

and the D-brane coordinate fields can be diagonalized simultaneously by a suitable gauge transformation. The corresponding eigenvalues $y_{a}^{i}, a=1, \ldots, N$ of the $Y^{i}$ then represent the collective transverse coordinates of the $N$ D-branes. In this limit, the parallel D-branes are very far apart from each other and massless vector states emerge only when fundamental strings start and end on the same D-brane. The gauge group is then $U(1)^{N}$. Since the energy of a string which stretches between two D-branes is

$$
M \propto \mathcal{T}\left|y_{a}-y_{b}\right|
$$

more massless vector states emerge when the branes very close to each other. The collection of all massless states corresponding to an elementary string starting and ending on either the same or a different D-brane forms a $U(N)$ multiplet. The offdiagonal components of the $Y^{i}$ and the remnant gauge fields describe the dynamics of the short open strings interacting with the branes, with Dirichlet boundary conditions. One obtains in this way an effective low-energy field theory of massless non-Abelian gauge excitations.

We now consider the interaction of such states with groups of $N^{\prime}$ D-particle defects that puncture the stack of $N$ parallel D-branes, as appropriate for a nonAbelian extension of the D-particle foam model discussed in the previous subsection: see Fig. 2, The analysis of [10] has demonstrated explicitly that the resulting dynamics of the massless excitations present during the recoil/capture stage is also described by a Born-Infeld lagrangian with $U\left(N^{\prime}\right)$ group. 
It is useful for our purposes to repeat briefly the technical details of the construction, so as to clarify the underlying physics. To describe the moduli-space dynamics of a multi-D-brane system, we use the construction described in [21, which leads to the clearest physical interpretation. In this picture, the assembly of D-branes, including all their elementary string interactions, is regarded as a composite 'fat brane' which couples to a single fundamental string with a matrix-valued coupling in a T-dual picture employing Neumann boundary conditions ${ }^{4}$. The resulting effective theory is described by a $\sigma$ model on a world-sheet with the 'effective' topology of a disc, propagating in the background of a non-abelian $U(N)$ Chan-Paton gauge field.

The relevant $U(N)$-invariant matrix $\sigma$-model action is

$$
\begin{gathered}
S_{N}[X ; A]=\frac{1}{4 \pi \alpha^{\prime}} \int_{\Sigma\left\{z_{a b}\right\}} d^{2} z \\
\operatorname{tr} \eta_{\mu \nu} \partial X^{\mu} \bar{\partial} X^{\nu}-\frac{1}{2 \pi \alpha^{\prime}} \oint_{\partial \Sigma\left\{z_{a b}\right\}} \operatorname{tr} Y_{i}\left(x^{0}(s)\right) d X^{i}(s) \\
+\oint_{\partial \Sigma\left\{z_{a b}\right\}} \operatorname{tr} A^{0}\left(x^{0}(s)\right) d X^{0}(s)
\end{gathered}
$$

where $\eta_{\mu \nu}$ is a (critical) flat $9+1$-dimensional space-time metric. The worldsheet fields $X, Y$ and $A$ are $N \times N$ Hermitian matrices transforming in the adjoint representation of $U(N)^{5}$. The traces in (19) are taken in the fundamental representation. The surface $\Sigma\left\{z_{a b}\right\}$ is a sphere with a set of marked points $z_{a b}, 1 \leq a, b \leq N$, on it. For each $a=b$ it has the topology of a disc $\Sigma$, while for each pair $a \neq b$ it has the topology of an annulus. The variable $s \in[0,1]$ parametrizes the circle $\partial \Sigma$. It was shown in 21] that the action (19) describes an assembly of $N$ parallel D-branes with fundamental oriented open strings stretching between each pair of them. The diagonal component $Y_{a a}$ of the matrix field $Y$ parametrizes the Dirichlet boundary condition on D-brane $a$, while the off-diagonal component $Y_{a b}=Y_{b a}^{*}$ represents the Dirichlet boundary condition for the fundamental oriented open string whose endpoints attach to D-branes $a$ and $b$. The matrix field $A^{0}$ parametrizes the usual Neumann boundary conditions in the temporal direction of the target space.

The action (19) is written in terms of Neumann boundary conditions on the configuration fields, which is the correct description of the dynamics of the D-branes in

\footnotetext{
${ }^{4}$ For subtleties in applying the T-dual picture, see 22, 23. We note here that one assumes 21 10. that the Neumann picture is the fundamental picture to describe the propagation of strings in fat-brane backgrounds. The Dirichlet picture is then derived by applying T-duality as a canonical functional integral transformation.

${ }^{5}$ We consider only the case of oriented open strings. For unoriented open strings, the global symmetry group $U(N)$ is replaced by $O(N)$ or $U S p(N / 2)$.
} 
this picture, but it is straightforward to apply a functional T-duality transformation on the fields of (19) to express it in the usual, equivalent Dirichlet parametrization 21. The configuration

$$
A^{\mu}=\left(A^{0},-\frac{1}{2 \pi \alpha^{\prime}} Y^{i}\right)
$$

can be interpreted as a $U(N)$ isospin gauge field dimensionally reduced to the worldline of the D-brane [9, 24].

As discussed in 10, 21, quantum fluctuations of the couplings $Y_{i}^{a b}$ provide infinitesimal separations between the $N$ constituent D-branes proportional to the string coupling $g_{s}$, and also allow for the endpoints of the fundamental strings to fluctuate in space-time. One must then integrate out all the fluctuations among the fat-brane constituents, i.e., over all of the marked points of $\Sigma\left\{z_{a b}\right\}$. This necessarily makes the action non-local. By $U(N)$ gauge invariance, the resulting $\sigma$-model partition function then becomes the expectation value, in a free (scalar) $\sigma$ model, of the path-ordered $U(N)$ Wilson-loop operator $W[\partial \Sigma ; A]$ along the boundary of the world-sheet disc $\Sigma$,

$$
\begin{aligned}
Z_{N}[A] & \equiv \sum_{\text {genera }} \int[d X] \int_{\Sigma} \prod_{a, b=1}^{N} d^{2} z_{a b} \mathrm{e}^{-S_{N}[X ; A]} \\
& \simeq\langle W[\partial \Sigma ; A]\rangle_{0} \equiv \int D x \mathrm{e}^{-N^{2} S_{0}[x]} \operatorname{tr} P \exp \left(i g_{s} \oint_{\partial \Sigma} A_{\mu}\left(x^{0}(s)\right) d x^{\mu}(s)\right),
\end{aligned}
$$

where $d X$ is the normalized invariant Haar measure for integration on the Lie algebra of $N \times N$ Hermitian matrices and

$$
S_{0}[x]=\frac{1}{4 \pi \alpha^{\prime}} \int_{\Sigma} d^{2} z \eta_{\mu \nu} \partial x^{\mu} \bar{\partial} x^{\nu}
$$

is the free $\sigma$-model action for the fundamental string. The path integral measure $D x$ is normalized so that $\langle 1\rangle_{0}=1$. The partition function (21) describes the dynamics of a fat brane, which is depicted by the system of parallel dashed-triple-dotted lines in Fig. 2 ,

The low-energy effective action for the D-brane configuration is now obtained by integrating out the fundamental string configurations $x$ in (21). To lowest order in the gauge-covariant derivative expansion, the result is given by a non-Abelian Born-Infeld effective action $Z_{N}[A] \simeq \mathrm{e}^{-N^{2} \Gamma_{\mathrm{NBI}}[A]}$, where

$$
\Gamma_{\mathrm{NBI}}[A]=\frac{c_{0}}{\sqrt{2 \pi \alpha^{\prime}} g_{s}} \int d t \operatorname{tr}(\operatorname{Sym}+i \zeta \mathrm{Asym})\left(\operatorname{det}_{\mu, \nu}\left[\eta_{\mu \nu} I_{N}+2 \pi \alpha^{\prime} g_{s}^{2} F_{\mu \nu}\right]\right)^{1 / 2}
$$


is the non-abelian Born-Infeld action for the dimensionally-reduced gauge field $A_{\mu}$. Here $c_{0}$ is a numerical constant and $t=x^{0}(s=0)$ is the world-sheet zero mode of the temporal embedding field, $I_{N}$ is the $N \times N$ identity matrix, Sym denotes the symmetrized matrix product

$$
\operatorname{Sym}\left(M_{1}, \ldots, M_{n}\right)=\frac{1}{n !} \sum_{\pi \in S_{n}} M_{\pi_{1}} \cdots M_{\pi_{n}}
$$

and Asym is the antisymmetrized matrix product

$$
\operatorname{Asym}\left(M_{1}, \ldots, M_{n}\right)=\frac{1}{n !} \sum_{\pi \in S_{n}}(\operatorname{sgn} \pi) M_{\pi_{1}} \cdots M_{\pi_{n}}
$$

The symmetric and antisymmetric products on functions $f\left(M_{1}, \ldots, M_{n}\right)$ of $n$ matrices $M_{k}$ are defined in [10. The symmetrization and antisymmetrization operations have the effect of removing the ambiguity in the definition of the space-time determinant in (23) for matrices with non-commuting entries.

The components of the field strength tensor in (23) are given by

$$
2 \pi \alpha^{\prime} F_{0 i}=\frac{d}{d t} Y_{i}-i g_{s}\left[A_{0}, Y_{i}\right] \quad, \quad\left(2 \pi \alpha^{\prime}\right)^{2} F_{i j}=g_{s}\left[Y_{i}, Y_{j}\right]
$$

and the constant $\zeta \in \mathbb{R}$ is left arbitrary so that it interpolates among proposals for the true trace structure inherent in the non-Abelian generalization of the BornInfeld action. The case $\zeta=0$ corresponds to the original proposal in [25], while the trace structure with $\zeta=1$ was suggested, in a different context, in [26]. The two-loop world-sheet $\beta$ function for the model (21) was calculated in 22 to be

$$
\beta_{i}^{a b} \equiv \frac{\partial Y_{i}^{a b}}{\partial \log \Lambda}=-\left(2 \pi \alpha^{\prime} g_{s}\right)^{2}\left(D^{\mu} F_{\mu i}\right)^{a b}+2\left(2 \pi \alpha^{\prime} g_{s}\right)^{3}\left(D^{\mu}\left[F_{\mu \nu}, F_{i}^{\nu}\right]\right)^{a b}+\mathcal{O}\left(\left(\alpha^{\prime} g_{s}\right)^{4}\right)
$$

where

$$
D_{0}=\frac{d}{d t}-i g_{s}\left[A_{0}, \cdot\right] \quad, \quad D_{i}=\frac{i g_{s}}{2 \pi \alpha^{\prime}}\left[Y_{i}, \cdot\right]
$$

are the components of the dimensionally-reduced gauge-covariant derivative. It is readily seen that (27) coincides with the variation of the action (23) with $\zeta=1$ up to the order indicated in (27), so that the world-sheet RGEs $\beta_{i}^{a b}=0$ coincide with the equations of motion of the D-branes. The first term in (27) yields the (reduced) Yang-Mills equations of motion, while the second term represents the first-order stringy correction to the Yang-Mills dynamics. 
If we now replace the space-time coordinates $Y_{i}$ and velocity $U_{i}$ used in the previous subsection by matrix quantities:

$$
Y_{i}\left(x^{0}\right)^{a b}=Y_{i}^{a b}+U_{i}^{a b} x^{0}
$$

the discussion of D-particle recoil in the previous subsection may be generalized to the D-brane model illustrated in Fig. 2, where the intersection is viewed as a foam model of virtual D-branes emerging from the vacuum. Because of this assumption, the D-particle defects are not real, but appear as virtual excitations of the foam, and as such are bound to carry the same quantum numbers as the vacuum.

However, a model where the bulk ten-dimensional space, in which the D-branes are embedded, is characterised by a given density of such D-particle defects, occasionally crossing the intersection, is also a viable model for space time foam. To an observer on the intersection, the crossings of the D-particle defects will appear as 'virtual' D-particle fluctuations in the vacuum. Formally, the (T-dual) version of the recoil operator describes an effective non-Abelian gauge potential of the form [10]:

$$
A_{0}^{a b}=0 ; \quad A_{i}^{a b}=\left(\epsilon Y_{i}^{a b}+U_{i}^{a b} x^{0}\right) \Theta\left(x^{0} ; \epsilon\right)
$$

where $a, b$ are a colour indices. As shown in 10, its dynamics is that of a nonAbelian Born-Infeld described by the lagrangian (23).

Thus, in analogy with the Abelian case of an isolated D-particle, the recoil and capture of string matter by groups of $N^{\prime}$ such defects results in gauge excitations transforming in the adjoint representation of the $U\left(N^{\prime}\right)$ group. For real defects, this group might not be connected with the original gauge group of the string excitations localised on the intersection. However, given that in our model of foam the Dparticles are viewed as virtual excitations of the ground state of our system, and we insist on the possibility of re-emission of the string matter into the (stacked) brane world after the capture stage, the two groups must be the same. In other words, the number $N^{\prime}$ of the constituent D-particles in each excitation of the non-Abelian foam has to be such that the resulting $U\left(N^{\prime}\right)$ group must be locally isomorphic to the $U\left(N_{1}\right) \otimes U\left(N_{2}\right) \otimes \ldots$ group coming from the the stacks of the intersecting Dbranes. This means that the gauge boson configurations (111) that emerge from the recoil/capture stage described in the previous subsection must here be thought of as parts of a superposition with the gauge boson excitations arising from open strings on the intersecting stack of D-branes, realizing the same gauge group.

It follows from these considerations that the above procedure describes only the interaction of massless gauge particles (and their superpartners), such as Standard Model photons and gluons, with the space-time foam. There is no possibility of 
a non-trivial interaction of electrically-charged massive particles, such as electrons, with the D-particle defects. As in the Abelian case, this has to do with the fact that such charged probes transform according to bi-fundamental representations of the $U\left(N_{1}\right) \otimes U\left(N_{2}\right) \ldots$ group, whilst the composite open-string/D-particle excitations transform in the adjoint representation, and hence behave like gauge particles. The D-particle foam medium is transparent to such elementary matter particles, because of the conservation of electric charge, which cannot be broken by the gravitational space-time foam. We stress that this picture of space-time foam is not covered by critical string theory arguments, because recoil operators (30) are ignored in such treatments. In our approach, it is the presence of such non-conformal, relevant operators that leads to the non-trivial non-equilibrium space-time foam effects for photons that we have examined here.

The above possible effects of non-Abelian gauge interactions of D-particle groups may be suppressed significantly for composite particles containing gluons, such as protons. As argued in [27, 28, propagation effects and energy uncertainties in collision processes due to the Liouville foam interactions of ultra-high-energy protons, might be responsible for the possible persistence of ultra-high-energy cosmic rays [29] beyond the expected GZK cutoff in the spectrum. However, as observed in 28, the parameter $\mathcal{M}$ needed to modify the proton dispersion relation would be of order $10^{-16}-10^{-17}$ smaller than the Planck mass, much smaller than the corresponding parameter (of order one in Planck units) that would be sufficient to remove the expected analogous cutoff for gamma rays above $20 \mathrm{TeV}[30]^{6}$. Such a difference might well be explained by the fact that only the gluons in the proton feel the effects of the D-particle foam. Since the characteristic scale of a QCD bound state suach as the proton is of order $\Lambda_{Q C D} \sim 200 \mathrm{GeV}$, there could be an extra suprression factor $\Lambda_{Q C D} / M_{P} \sim 10^{-19}$ multiplying the coefficient of $E / \mathcal{M}$ in the modification of the proton dispersion relations. However, more work is required before firm conclusions can be reached regarding such issues in the context of the model of [5]. Nevertheless, the above analysis makes it clear that details in the dynamics of the interactions between foam and matter can make a crucial difference from naive generic phenomenological analyses, which could be misleading.

\footnotetext{
${ }^{6}$ Some doubt [30, 31, 32 has been expressed on whether a TeV-infrared background crisis exists. This is due to ambiguities in measurements of extragalactic light [32], as well as theoretical uncertainties in the intrinsic model for generation of $\mathrm{TeV}$ photons in Active Galactic Nuclei. In our view, observations of photons in the $100-200 \mathrm{GeV}$ energy range, as well as $\mathrm{TeV}$ sources at different redshifts, are needed in order to constraint seriously space time foam models with modified photon dispersion relations.
} 


\section{Discussion}

In light of the analysis in the present work, it may still be that the study of the arrival times of photons in gamma-ray bursts (GRBs) [33] is the best experimental test, to date, for probing possible quantum-gravitational effects on particle propagation, through their high sensitivity to any possible refractive index for photons propagating in vacuo.

We have pointed out previously that ultra-high-energy neutrino emission from GRBs would also be very sensitive to any analogous effects on neutrino propagation [18. However, in the type of intersecting-brane model studied here, neutrinos are considered as chiral fermions that transform in bi-fundamental representations of the weak-interaction gauge group $S U(2)_{L} \otimes S U(2)_{R}$. As such, they do not interact, at least at tree level, with D-particles. However, this conclusion, stemming from the analysis of [10] described above, applies only to the case in which the open string is attached with both ends on the D-particle, and hence the open strings have double Dirichlet (DD) boundary conditions.

This raises the question whether, in principle, one may consider the recoil of strings with one end attached by a Dirichlet boundary condition to the D-particle and the other attaches freely with a Neumann boundary condition to intersecting Dbranes, as shown in Fig. 2. If such ND strings are consistent string configurations, as argued in [16, the earlier discussion about the lack of Lorentz violation for charged particles such as electrons would be unaffected, though one could apply the ND formalism to neutrinos. However, in our view there is a issue in defining such configurations, associated with the issue of conformal invariance for pinched highergenus configurations, as illustrated in Fig. 3. If such open ND strings existed, there is a question whether they could be consistent configurations, from a target space-time point of view. Because of the mixed ND boundary conditions, when one pinches a higher-genus world-sheet graph, the pinch would contain a world-sheet strip with infinite length and vanishing width, on the ends of which one would impose two different boundary conditions. This would require the introduction of a non-local phase-space operator, called a 'zipper' in [16]. It remains to be established whether this is a consistent local operator in conformal field theory.

Another important issue concerns higher-order self-energy graphs. The naive (local field theory) point of view would be that the quantum fluctuations of open strings interacting with D-particles are described by annulus or higher-topology world-sheet graphs, involving the exchange of all string states around the loop. In principle, some such states might interact with the D-particle. However the same argument based on conformal invariance that was given above for pinched tree-level 


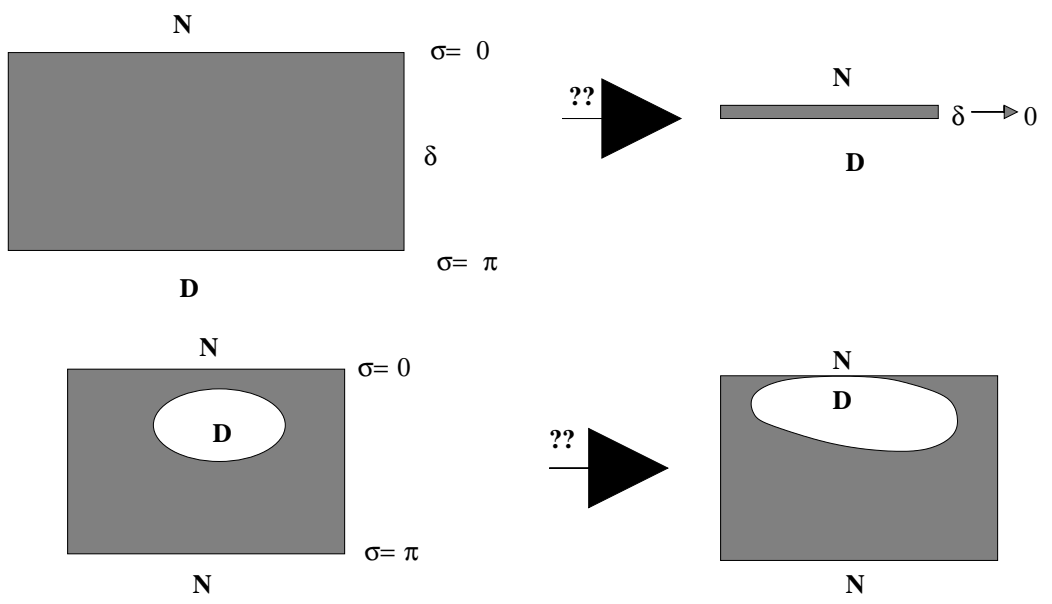

Figure 3: Top drawing: if ND open strings were consistent conformal invariant backgrounds, then the pinching of world-sheet surfaces should be allowed. However, this would require the superimposition of different types of boundary conditions, which is impossible. This argument also rules out the appearance of mixed boundary conditions for open strings exchanged in string loops, as seen in the bottom drawing. Quantum string higher-genus corrections to ND strings are inadmissible, as pinched genera in the loop would also require a superimposition of two different types of boundary conditions in the limit where the world-sheet strip width vanishes.

world sheets also applies here. This can be seen, in particular, by considering the lower part of Fig. 3, featuring a chiral fermion with Neumann boundary conditions and a string loop with Dirichlet boundary conditions, representing quantum corrections in which a D-particle interacts with the virtual string state. We conclude that the non-interaction of chiral fermions with the D-particle foam is an exact result, valid to all orders in the string genus expansion.

However, as discussed above, gauge fields may in general interact with the spacetime foam, leading to violations of Lorentz invariance that are non-universal and generating violations of the equivalence principle at high energies that may be probed by astrophysical observations. 


\section{Acknowledgements}

We would like to thank H. Hofer for his interest and support. The work of N.E.M. is partially supported by the European Union through contract HPRN-CT-200000152. The work of D.V.N. is supported by D.O.E. grant DE-FG03-95-ER-40917.

\section{References}

[1] J. D. Bekenstein, Phys. Rev. D 7 (1973) 2333; S. W. Hawking, Commun. Math. Phys. 43 (1975) 199 and S. W. Hawking, Phys. Rev. D 13 (1976) 191.

[2] S. W. Hawking, Commun. Math. Phys. 87 (1982) 395; J. R. Ellis, J. S. Hagelin, D. V. Nanopoulos and M. Srednicki, Nucl. Phys. B 241 (1984) 381; J. R. Ellis, N. E. Mavromatos and D. V. Nanopoulos, Phys. Lett. B 293 (1992) 37 arXiv:hep-th/9207103.

[3] J. R. Ellis, N. E. Mavromatos, D. V. Nanopoulos and A. S. Sakharov, arXiv:astro-ph/0309144

[4] J. R. Ellis, N. E. Mavromatos and A. S. Sakharov, arXiv:astro-ph/0308403.

[5] J. R. Ellis, N. E. Mavromatos and D. V. Nanopoulos, Phys. Rev. D 61 (2000) 027503 arXiv:gr-qc/9906029; Phys. Rev. D 62 (2000) 084019 arXiv:gr-qc/0006004.

[6] G. Amelino-Camelia, J. R. Ellis, N. E. Mavromatos and D. V. Nanopoulos, Int. J. Mod. Phys. A 12 (1997) 607 arXiv:hep-th/9605211.

[7] G. Amelino-Camelia, J. R. Ellis, N. E. Mavromatos, D. V. Nanopoulos and S. Sarkar, Nature 393 (1998) 763 arXiv:astro-ph/9712103.

[8] T. Jacobson, S. Liberati and D. Mattingly, arXiv:astro-ph/0212190; arXiv:gr-qc/0303001.

[9] J. Polchinski, Phys. Rev. D50 (1994) 6041; Phys. Rev. Lett. 75 (1995) 184; TASI Lectures on D-branes, arXiv:hep-th/9611050; J. Polchinski, S. Chaudhuri and C. Johnson, Notes on D-branes, hep-th/9602052

C.P. Bachas, Lectures on D-branes, hep-th/9806199.

[10] N. E. Mavromatos and R. J. Szabo, Phys. Rev. D 59 (1999) 104018 arXiv:hep-th/9808124. 
[11] I.I. Kogan and N.E. Mavromatos, Phys. Lett. B375 (1996) 111.

[12] V. Periwal and O. Tafjord, Phys. Rev. D54 (1996) 4690.

[13] I.I. Kogan, N.E. Mavromatos and J.F. Wheater, Phys. Lett. B387 (1996) 483.

[14] V. Gurarie, Nucl. Phys. B410 (1993) 535;

M.A.I. Flohr, Int. J. Mod. Phys. A11 (1996) 4147; A12 (1997) 1943;

M.R. Gaberdiel and H.G. Kausch, Nucl. Phys. B489 (1996) 293; Phys. Lett. B386 (1996) 131;

F. Rohsiepe, On Reducible but Indecomposable Representations of the Virasoro Algebra, hep-th/9611160;

I.I. Kogan, A. Lewis and O.A. Soloviev, Int. J. Mod. Phys. A13 (1998) 1345.

[15] J. Ellis, N.E. Mavromatos and D.V. Nanopoulos, Int. J. Mod. Phys. A12 (1997) 2639.

[16] I. I. Kogan and J. F. Wheater, Phys. Lett. B 403 (1997) 31 arXiv:hep-th/9703141.

[17] A. Strominger, Phys. Lett. B 383 (1996) 44 arXiv:hep-th/9512059; O. Bergman, M. R. Gaberdiel and G. Lifschytz, Nucl. Phys. B 509 (1998) 194 arXiv:hep-th/9705130.

[18] J. R. Ellis, N. E. Mavromatos, D. V. Nanopoulos and G. Volkov, Gen. Rel. Grav. 32 (2000) 1777 arXiv:gr-qc/9911055.

[19] E. Witten, Nucl. Phys. B460 (1996) 335.

[20] M. Berkooz, M. R. Douglas and R. G. Leigh, Nucl. Phys. B 480 (1996) 265 arXiv:hep-th/9606139.

[21] F. Lizzi, N.E. Mavromatos and R.J. Szabo, Mod. Phys. Lett. A13 (1998) 829.

[22] D. Brecher and M.J. Perry, Bound States of D-branes and the Non-abelian Born-Infeld Action, hep-th/9801127, to appear in Nucl. Phys. B.

[23] H. Dorn, Nonabelian Gauge Field Dynamics on Matrix D-branes in Curved Space and Two-dimensional $\sigma$-models, hep-th/9712057; J. High Energy Phys. 04 (1998) 013. 
[24] W. Taylor, Phys. Lett. B394 (1997) 283;

I.I. Kogan, G.W. Semenoff and R.J. Szabo, Mod. Phys. Lett. A12 (1997) 183.

[25] A.A. Tseytlin, Nucl. Phys. B501 (1997) 41.

[26] P.C. Argyres and C.R. Nappi, Nucl. Phys. B330 (1990) 151.

[27] Y. J. Ng, D. S. Lee, M. C. Oh and H. van Dam, Phys. Lett. B 507 (2001) 236 arXiv:hep-ph/0010152.

[28] J. R. Ellis, N. E. Mavromatos and D. V. Nanopoulos, Phys. Rev. D 63 (2001) 124025 arXiv:hep-th/0012216.

[29] G. Amelino-Camelia and T. Piran, Phys. Rev. D 64 (2001) 036005 arXiv:astro-ph/0008107.

[30] R. J. Protheroe and H. Meyer, Phys. Lett. B 493 (2000) 1 arXiv:astro-ph/0005349.

[31] F. W. Stecker, New Astron. Rev. 48 (2004) 437 arXiv:astro-ph/0304527;

F. W. Stecker, Astropart. Phys. 20 (2003) 85 arXiv:astro-ph/0308214.

[32] E. Dwek and F. Krennrich, arXiv:astro-ph/0406565

[33] J. R. Ellis, K. Farakos, N. E. Mavromatos, V. A. Mitsou and D. V. Nanopoulos, Astrophys. J. 535, 139 (2000) arXiv:astro-ph/9907340; J. R. Ellis, N. E. Mavromatos, D. V. Nanopoulos and A. S. Sakharov, Astron. Astrophys. 402, 409 (2003) arXiv:astro-ph/0210124. 